\title{
mesure de la profondeur des fondations par micro sismique transparence
}

\author{
par \\ J. Hurtado \\ Ingénieur Civil des Ponts et Chaussées \\ Sols et Structures
}

Il existe de nombreux cas où la connaissance de la profondeur ad'une fondation en place est nécessaire. En voici quelques exemples:

- La construction de sous-sols profonds en site urbain implique la connaissance des fondations voisines. Lorsqu'une construction proche d'une paroi moulée est fondée sur pieux il convient de connaître avec précision la position de la pointe des pieux pour évaluer les efforts induits sur la paroi.

- La surveillance des chantiers de fondations profondes nécessite en principe la présence permanente sur le chantier d'un surveillant connaissant les méthodes d'exécution utilisées. Le coût d'une telle surveillance est élevé. Une surveillance par échantillonnage, moins coûteuse, peut être utilement complétée par la mesure, en fin de chantier, de la profondeur de quelques éléments de la fondation pris au hasard.

- L'expertise judiciaire d'un sinistre de fondations pourrait souvent être mieux étayée par la vérification des profondeurs des éléments de fondation fournies par les documents tels qu'attachements ou plans des fondations.

Le seul moyen existant pour mesurer la profondeur des fondations d'un édifice construit était, récemment encore, lexécution de puits de visite. Cette méthode très coûteuse ne peut être mise en œuvre qu'avec précaution, notamment lorsque la base des fondations est sous le niveau de la nappe phréatique.

La Micro Sismique Transparence permet aujourd'hui de résoudre ce problème économiquement et sans diminuer la sécurité de la fondation.

\section{Généralités}

Soit une fondation $F$ de profondeur inconnue $h$. La fondation $\mathrm{F}$ peut être porteuse d'une structure $\mathrm{S}$.

La profondeur $\mathrm{h}$ peut être mesurée par Micro Sismique transparence.

Le procédé consiste à exécuter un forage parallèle à la fondation, à une distance $b$ aussi petite que possible de celle-ci, et de profondeur supérieure de quelques mètres à la profondeur présumée hp de la fondation F.

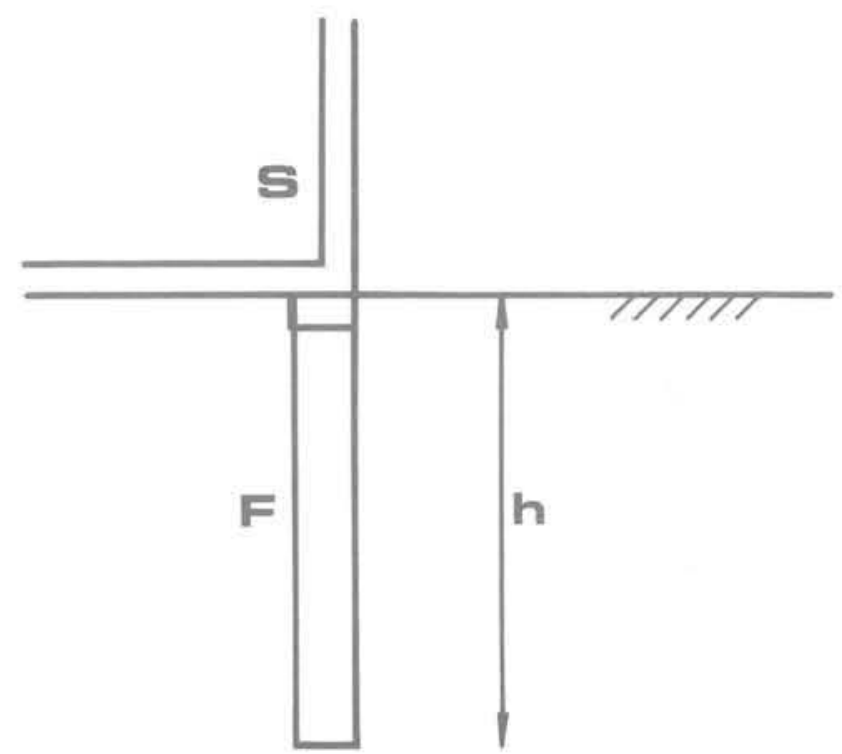

Fig. 1 Exemple de fondation à reconnaître 
Le forage est équipé d'un tube P.V.C. $\varnothing 60 \mathrm{~mm}$ sur toute sa hauteur. II est maintenu plein d'eau pendant les mesures. On choisit un point $C$ de la structure $S$ ou de la partie supérieure de la fondation $F$. En ce point on provoque un ébranlement (choc) à l'instant to L'ébranlement est percu au point $\mathrm{R}$ du forage à l'instant $\mathrm{t}_{1}$ à l'aide d'un récepteur, géophone ou similaire. On mesure le temps $t=t_{1}-t_{0}$ correspondant à la profondeur $r$ du point $R$.

On recommence la mesure en plusieurs points $R$ de façon à pouvoir tracer la courbe représentative de la fonction $t(r)$.

\section{2 Étude de la fonction $t(r)$}

Dans le cas simple où la vitesse de l'ébranlement est constante et égaie à $V_{1}$ dans la fondation $F$, constante et égale à $V_{2}$ dans le sol entre $\mathrm{F}$ et le forage, l'étude théorique de la fonction $t(r)$ conduit au développement suivant:

\subsection{Les trois expressions de $t(r)$}

Le trajet de l'ébranlement, de C en R, comprend deux parties :

$C P$ dans la fondation, à la vitesse $V_{1}>V_{2}$

$P R$ dans le sol, à la vitesse $V_{2}$

soient $x$ la profondeur de $P$.

b la distance du forage à la fondation

$$
\begin{aligned}
& t=\frac{x}{V_{1}}+\frac{\sqrt{b^{2}+(r-x)^{2}}}{V_{2}} \\
& \frac{d t}{d x}=0 \quad \frac{r-x}{\sqrt{b^{2}+(r-x)^{2}}}=\frac{V_{2}}{V_{1}}=\alpha
\end{aligned}
$$

posons $e=r-x$

$$
e=b \frac{\alpha}{\sqrt{1-\alpha^{2}}}
$$

La différence des profondeurs de $\mathrm{P}$ et $\mathrm{R}$ est indépendante de $r$.

Les trois expressions de $t(r)$ sont

$$
\begin{array}{ll}
r \leqslant e & t=\frac{\sqrt{r^{2}+b^{2}}}{V_{2}} \\
e \leqslant r \leqslant h+e & t=\frac{r-e}{V_{1}}+\frac{\sqrt{b^{2}+e^{2}}}{V_{2}} \\
h+e \leqslant r & t=\frac{h}{V_{1}}+\frac{\sqrt{(r-h)^{2}+b^{2}}}{V_{2}}
\end{array}
$$

- La première expression n'a pas d'intérêt pratique.

- La deuxième expression est une fonction linéaire $\mathrm{t}=\frac{\mathrm{r}}{V_{1}}+\mathrm{B}$

- La troisième expression est une fonction qui admet une asymptote $A$. Cette asymptote coupe la droite $t=\frac{r}{V_{1}}$ en un point d'abscisse $r=h$, quels que soient $b, V_{1}$ et $V_{2}$

\subsection{Interprétation du diagramme $t(r)$}

Dans le cas simple défini ci-dessus l'interprétation graphique du diagramme $t(r)$ est faite de la façon suivante :

- On trace une droite " moyenne " passant par les points du diagramme $t(r)$ pour $r<h_{p}$.

La pente de cette droite est $\frac{1}{V}$.

- On trace une parallèle $D$ à cette droite passant par l'origine.

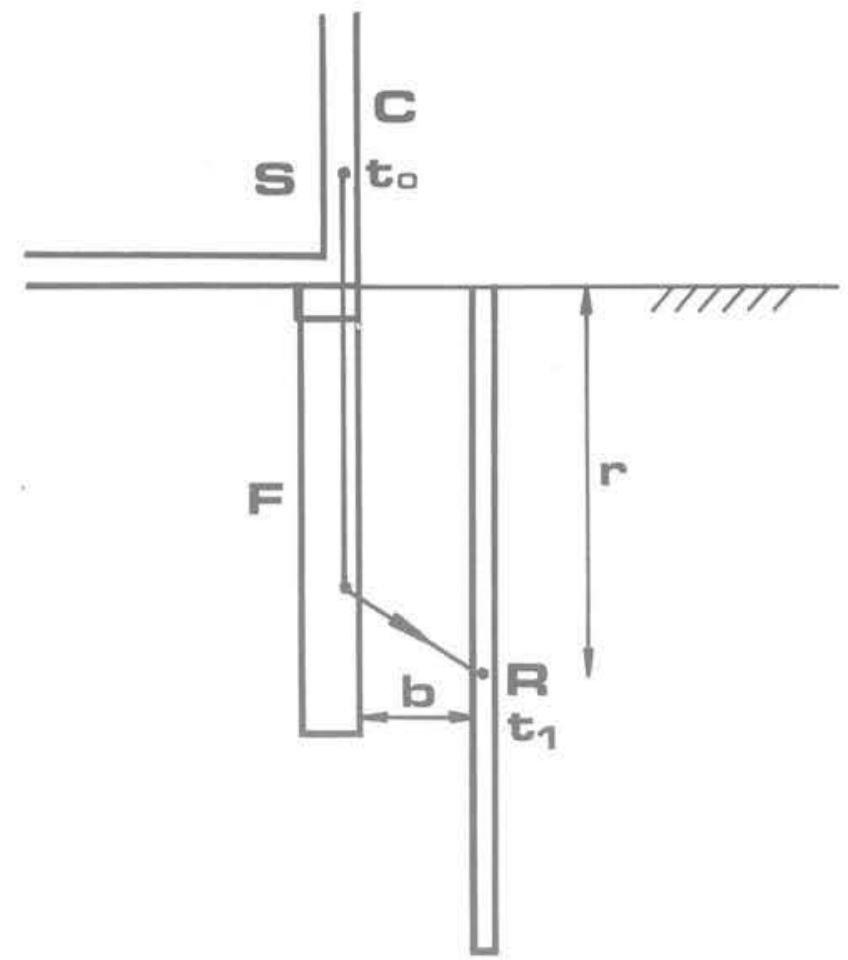

Fig. 2 Principe de la mesure

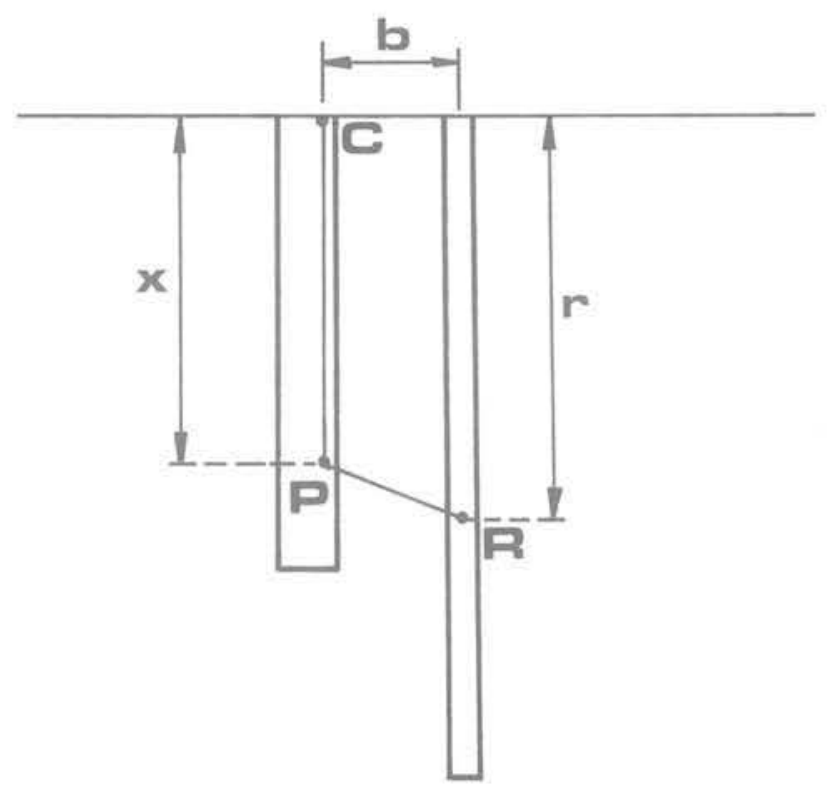

Fig. 3 Principe du calcul de $t(r)$ 


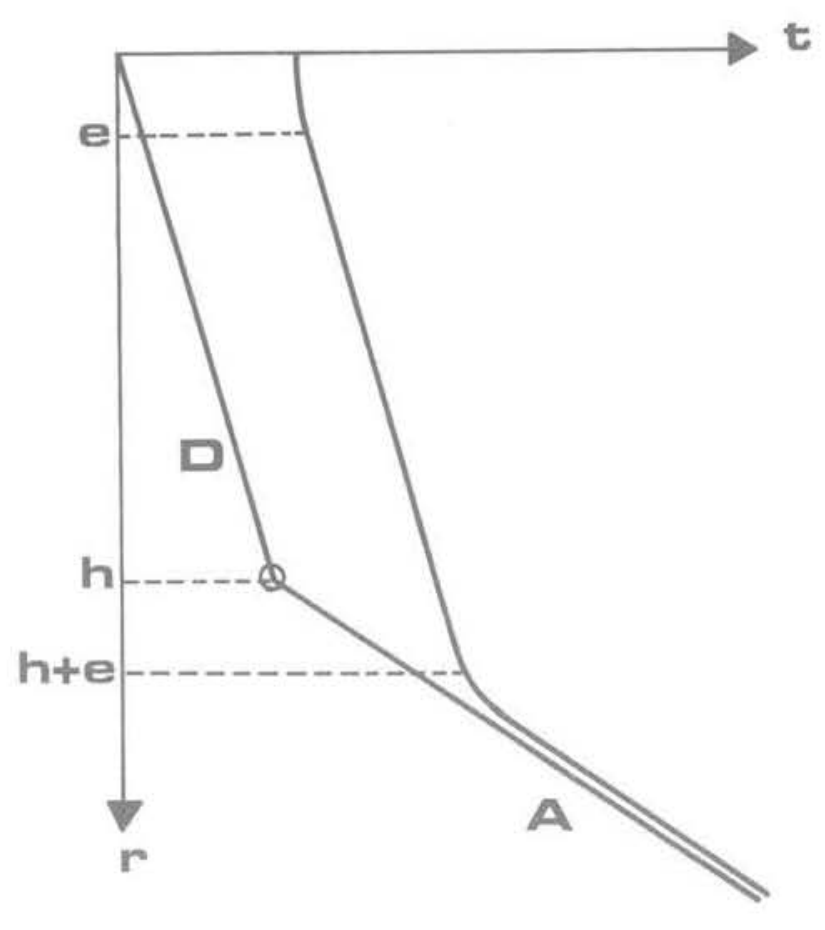

Fig. 4 Exemple de fonction $t(r)$

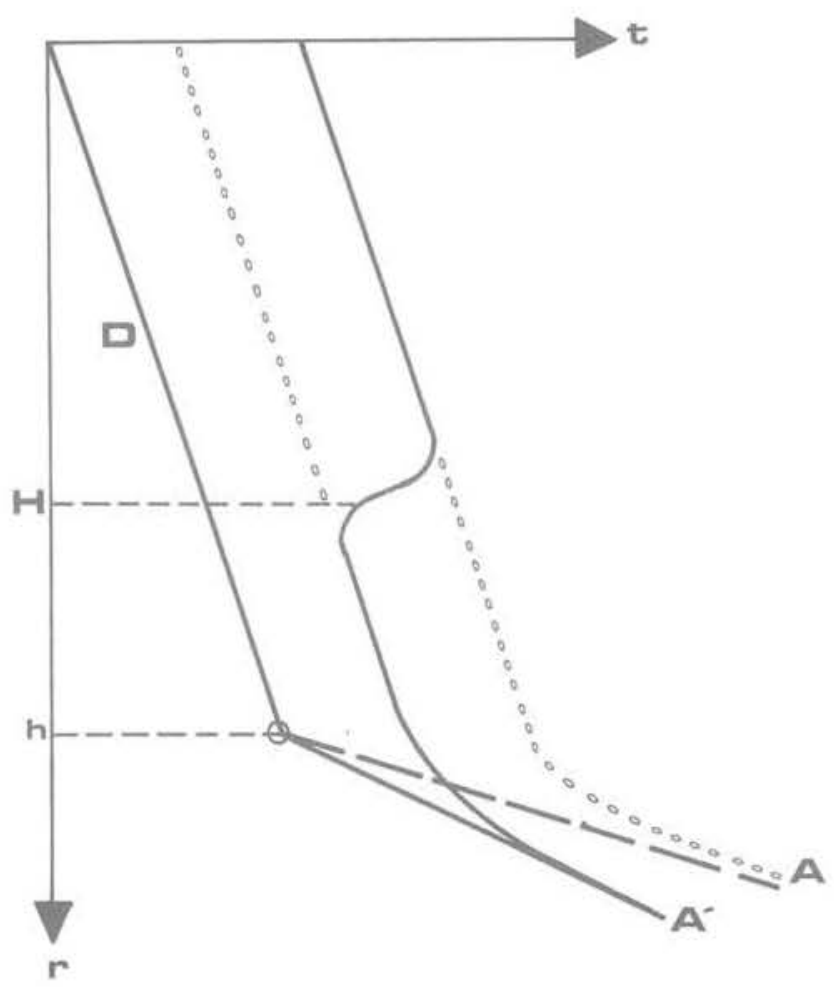

Fig. 6 Courbe $t(r)$ pour un sol hétérogène $(H<h)$

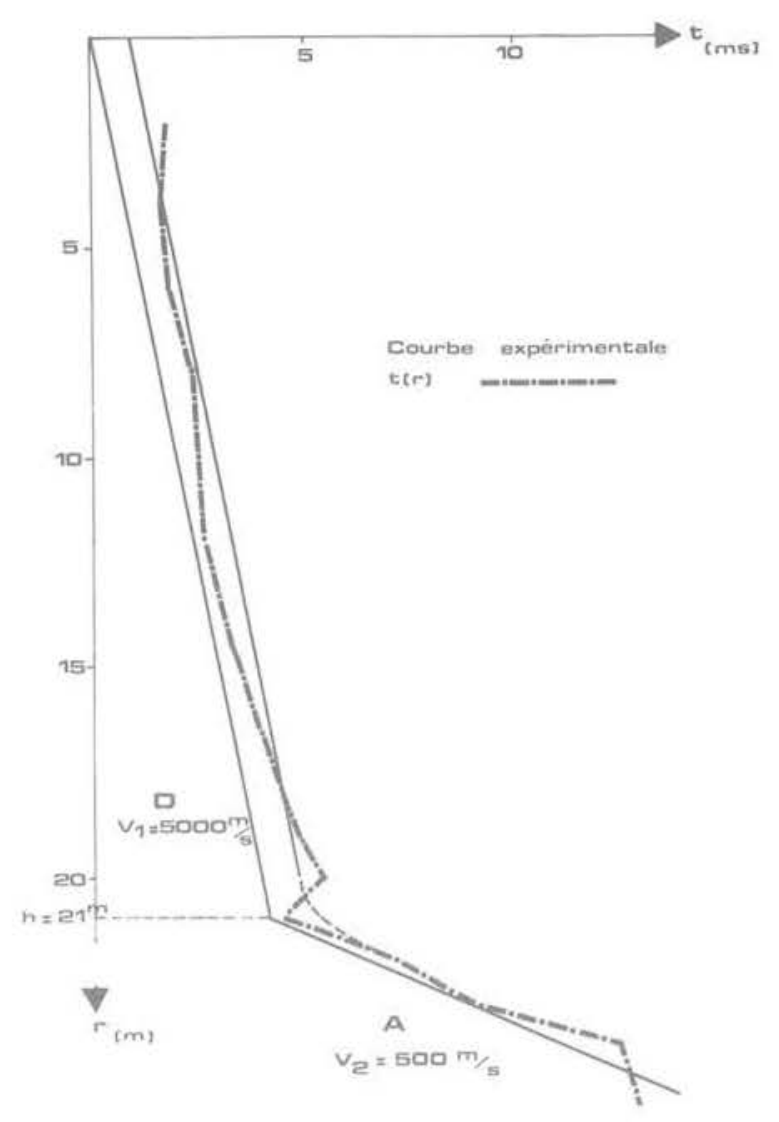

Fig. 5 Courbe expérimentale en terrain homogène

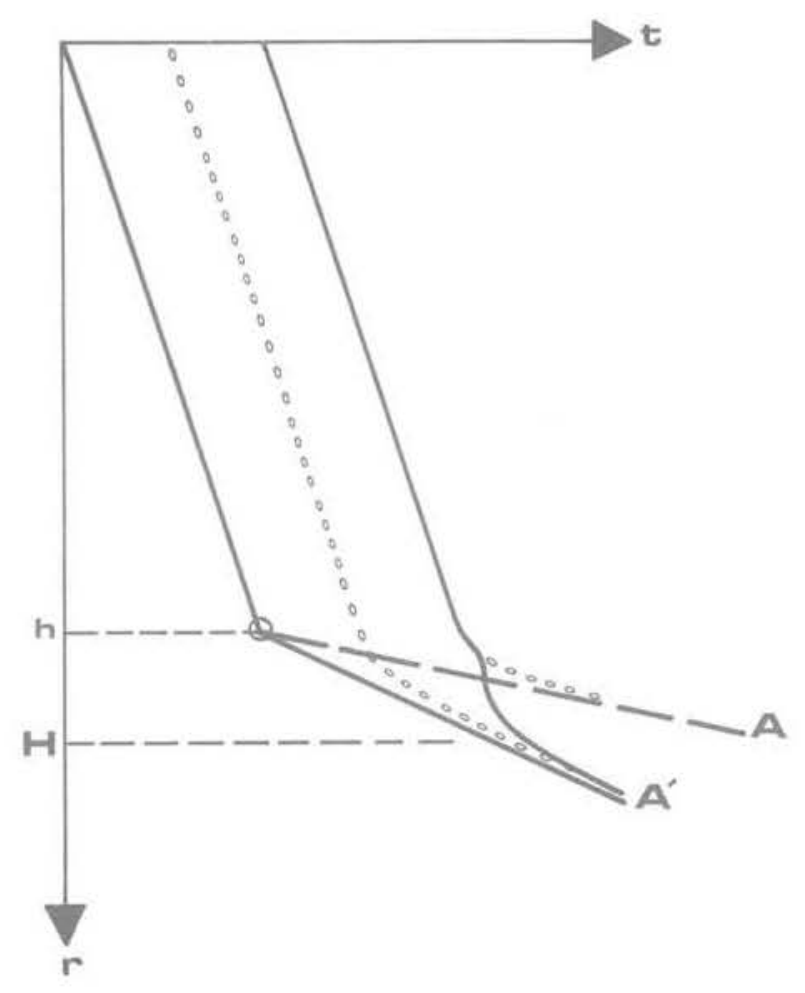

Fig. 7 Courbe $t(r)$ pour un sol hétérogène $(H>h)$ 


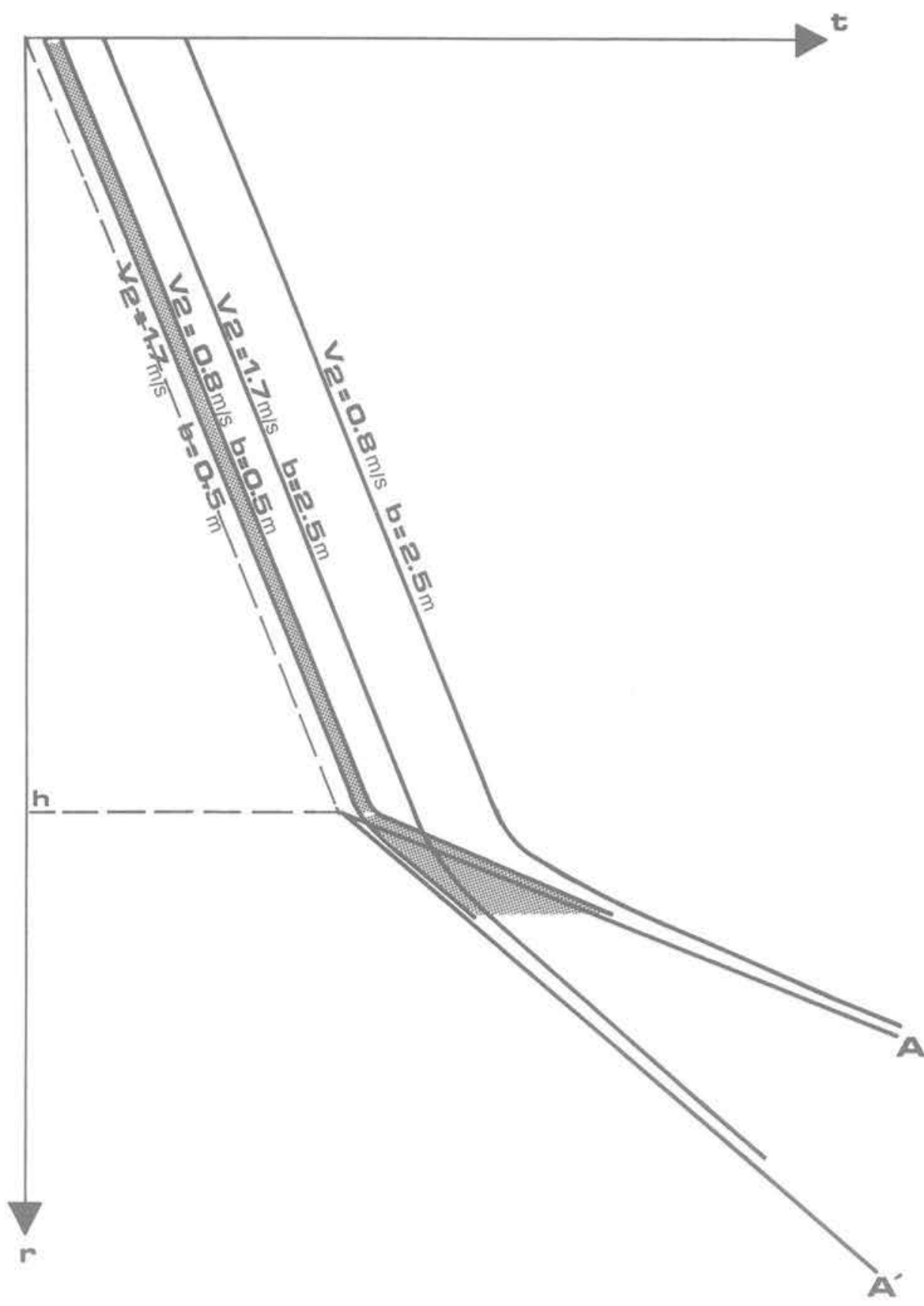

Fig. 8 Influence de la distance forage-fondation 


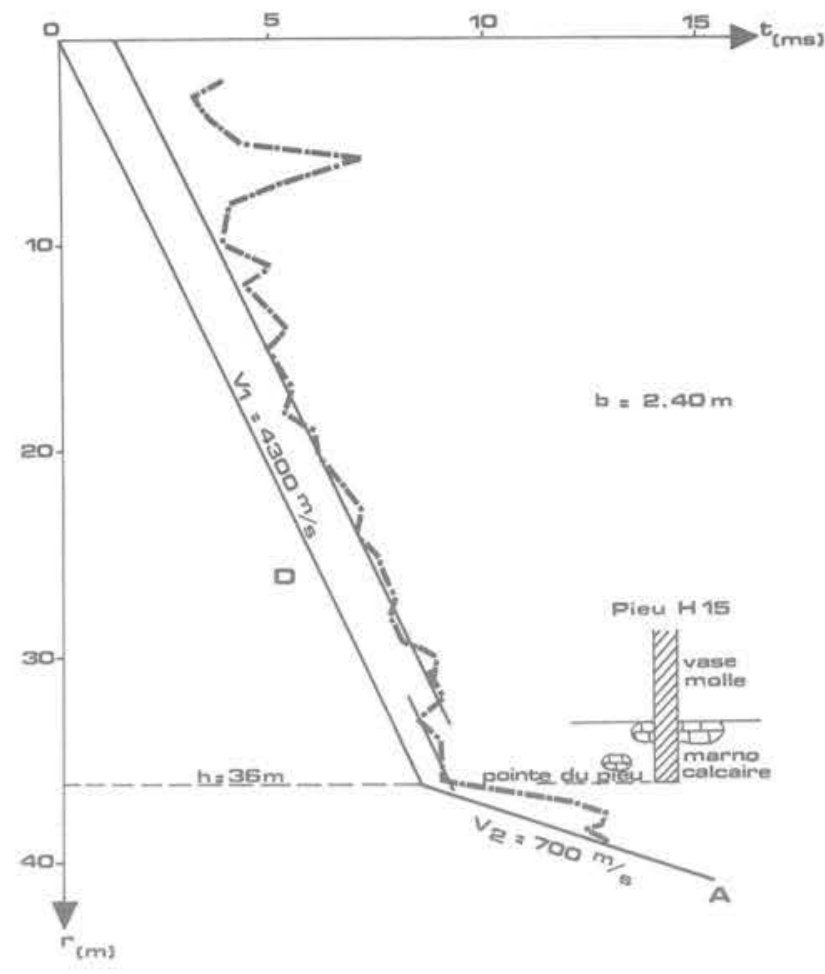

Fig. 9 1er exemple d'application

- On trace la droite "moyenne» A joignant les derniers points du diagramme $t(r)$

Cette droite est assimilée à l'asymptote de la fonction $t(r)$ : il est donc important que le forage ait une profondeur supérieure de plusieurs mètres à celle de la fondation, et cette surprofondeur doit être d'autant plus grande que le forage est éloigné de la fondation.

- Les droites A et D se coupent en un point d'abscisse $h$ qui est la grandeur cherchée.

Exemple: la figure 5 résume l'interprétation des mesures faites sur un chantier.

\subsection{Influence de la variation de la vitesse $V_{2}$}

Nous avons étudié jusqu'ici le cas simple où la vitesse de l'ébranlement dans le sol, $V_{2}$ était une constante.

Supposons maintenant que, par suite du changement de la nature du sol, catte vitesse devienne $V_{2}^{\prime}>V_{2}$ à partir de la profondeur $\mathrm{H}$.

Sur les figures 6 et 7 nous avons tracé les courbes $t(r)$ pour $V_{2}$ et $V_{2}$. Elles admettent les asymptotes $A$ et $A^{\prime}$.

La figure 6 correspond au cas $\mathrm{H}<\mathrm{h}$. Le raccordement des courbes se produit au-dessus de la base de la fondation. La difficulté d'interprétation tient au calcul de la pente de la droite $D$.

La figure 7 correspond au cas $\mathrm{H}>\mathrm{h}$. Le raccordement des courbes se produit au niveau de la base de la fondation. Pour que l'interprétation soit aisée, une surprofondeur du forage de mesure est nécessaire.

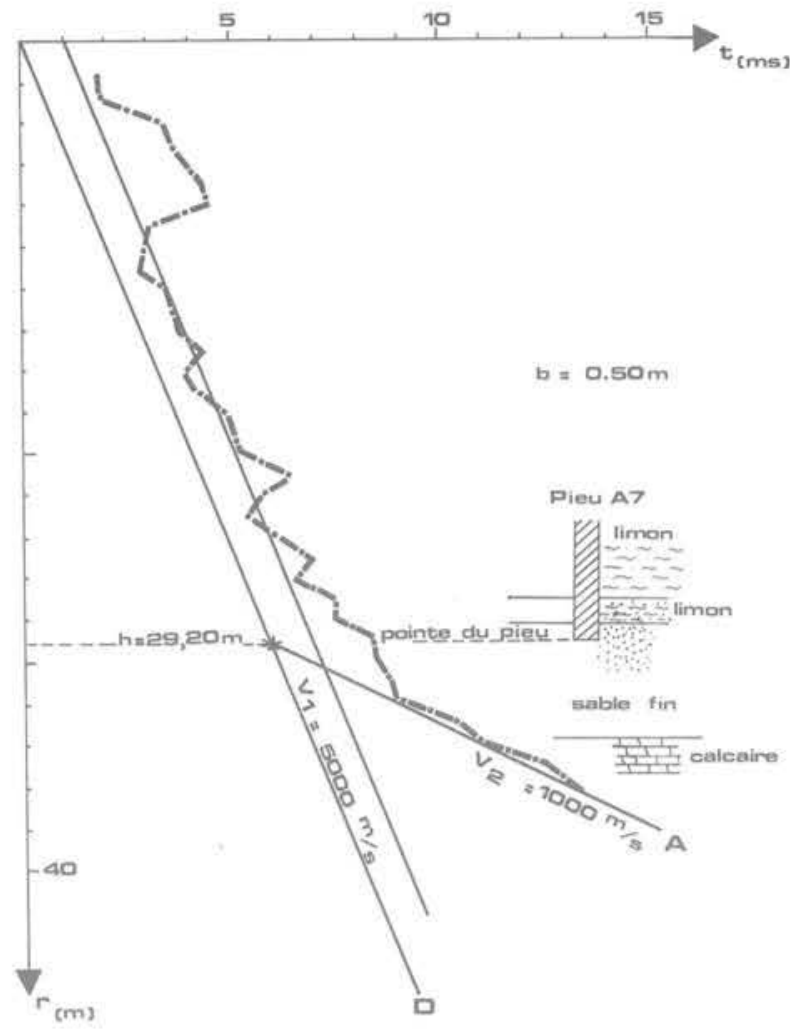

Fig. 10 2ème exemple d'application

2.4 Influence de la distance séparant le forage de la fondation

Sur la figure 8 sont reportées quatre courbes $t(r)$ théoriques pour $\mathrm{h}=30 \mathrm{~m}$ et $\mathrm{V}_{1}=5000 \mathrm{~m} / \mathrm{s}$.

$$
\begin{array}{ll}
b=0,5 \mathrm{~m} & V_{2}=800 \mathrm{~m} / \mathrm{s} \\
b=2,5 \mathrm{~m} & V_{2}=1700 \mathrm{~m} / \mathrm{s} \\
& V_{2}=800 \mathrm{~m} / \mathrm{s} \\
& V_{2}=1700 \mathrm{~m} / \mathrm{s}
\end{array}
$$

Si la vitesse du son dans le sol varie entre $800 \mathrm{~m} / \mathrm{s}$ et $1700 \mathrm{~m} / \mathrm{s}$ la courbe réelle enregistrée sera située dans les zones hachurées selon que $b=0,5 \mathrm{~m}$ ou $2,5 \mathrm{~m}$.

La surprofondeur du forage de mesure doit être de l'ordre de $8 \mathrm{~b}$ pour que l'interprétation soit suffisamment précise (connaissance de $\mathrm{h}$ à $\pm 20 \mathrm{~cm}$ près).

\section{Exemple d'interprétation pour un bâtiment fondé sur pieux}

Deux sondages de reconnaissance $F 1$ et $F 3$ ont été réalisés respectivement à $2,40 \mathrm{~m}$ et $0,50 \mathrm{~m}$ de deux pieux notés $\mathrm{H} 15$ et $\mathrm{A} 7$.

Ces sondages ont été équipés de tubes P.V.C. de façon à permettre la mesure de la longueur des pieux par le procédé M.S.T. Les résultats obtenus sont reportés sur les figures 9 et 10 qui indiquent clairement la précision et la qualité de la méthode. 
